\title{
Meningitis tuberculosa en adultos: Análisis de 53 casos
}

\author{
Margarita Enberg G., M. De la Luz Quezada B., Carolina de Toro V. y Luzmaría Fuenzalida L.
}

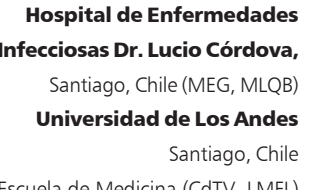

Escuela de Medicina (CdTV, LMFL)

Recibido: 29 agosto 2005 Aceptado: 27 enero 2006

Correspondencia a: Margarita Enberg Gaete docenberg@yahoo.com

\section{Tuberculous meningitis in adults: review of 53 cases}

Tuberculous Meningitis (TBM) is the most severe form of extrapulmonary tuberculosis. The clinical spectrum is broad and may be non-specific making early diagnosis difficult. This increases the incidence of mortality. We decribe the clinical characteristics of patients with TBM in the Dr. Lucio Córdova's Infectious Disease Hospital in Santiago, Chile, between 1995 and 2002. We review 53 adult cases of TBM, with a median age of 39 years. At admission $66 \%$ of the patients had some mental status deterioration, and the classic triad of symptoms of meningeal irritation was present only in $30 \%$. The cerebrospinal fluid (CSF) examination showed increased protein level, low glucose level and lymphocytic pleocytosis in most. Thirty percent of the patients were coinfected with HIV. The mortality in the later group was greater than in the TBM population as a whole (31 vs 17\%). TBM is still a present diagnostic problem, in spite of the new diagnostic methods. A high index of suspicion is required in order to make an early diagnosis.

Key words: Tuberculous meningitis, tuberculosis, aseptic meningitis, adults.

Palabras claves: Meningitis tuberculosa, tuberculosis, meningitis linfocitaria, adultos.

\section{Introducción}

$\mathrm{L}$ a meningitis tuberculosa (MTBC) es la inflamación de las leptomeninges por Mycobacterium tuberculosis y la forma más grave de tuberculosis (TBC) extrapulmonar ${ }^{3}$. La inespecificidad de sus síntomas, la lentitud en el diagnóstico etiológico y las secuelas neurológicas que puede producir, hacen de esta enfermedad un problema importante de salud, tanto en países desarrollados como en países en desarrollo 4,5 .

Cerca de 2.000 millones de personas en el mundo están infectadas por M. tuberculosis y sólo $10 \%$ desarrollará la enfermedad. Un determinante de esta baja tasa de enfermedad en infectados es la vacunación $\mathrm{BCG}$ en niños de países con alta prevalencia de $\mathrm{TBC}^{6}$.

Antes de la aparición del VIH, el principal factor favorecedor para desarrollar MTBC era la menor edad. Actualmente la co-infección con VIH es el principal factor, aumentando hasta 100 veces el riesgo de desarrollar TBC; se produce un pico entre los 20 y 49 años de edad, con un máximo de expresión entre los 35 y 39 años?. También asociado a la infección por VIH se ha producido un incremento de las formas extrapulmonares, en particular de MTBC. Este riesgo aumenta en proporción a la disminución del recuento de linfocitos CD4 $4^{8,9}$.
El pronóstico de la MTBC está influenciado por varios factores: la edad-mayor letalidad bajo los 5 años de edad y sobre los 50 años-, la duración de los síntomas, especialmente si la evolución del cuadro clínico es mayor a dos meses; la intensidad del déficit neurológico, y la tardanza en el inicio del tratamiento específico $^{2}$.

Los métodos de diagnóstico tradicionales -baciloscopia y cultivo- no contribuyen al diagnóstico precoz, debido a la baja sensibilidad de la tinción de Ziehl Nielsen y al lento crecimiento del microorganismo ${ }^{10}$. A pesar del avance de las técnicas de neuroimágenes y métodos de laboratorio, como la RPC y la serología (ELISA) para anticuerpos específicos, el diagnóstico de MTBC continúa siendo difícil ${ }^{4}$.

El objetivo de este trabajo es describir las características clínicas y de laboratorio, el tratamiento y evolución de la MTBC observadas en nuestra experiencia.

\section{Material y Método}

En un diseño observacional y descriptivo se analizaron retrospectivamente las historias clínicas de pacientes estudiados en el Hospital de Enfermedades Infecciosas Dr. Lucio Córdova, Centro Nacional de Referencia para MTBC, entre los años 1995 y 2002. 
Se incluyeron los pacientes con MTBC confirmada y los con alta sospecha de este diagnóstico.

Definiciones. MTBC confirmada: el caso con bacteriología positiva (baciloscopia o cultivo de Koch) o biopsia positiva (infiltración de los tejidos por Mycobacterium tuberculosis constatado por médico anátomopatólogo). Alta sospecha: cuadro clínico compatible con MTBC (cefalea, signos meníngeos, compromiso de conciencia), evidencia clínica o radiológica de TBC en otros órganos o historia previa de TBC, asociado a parámetros citoquímicos del LCR característicos de TBC (proteínas $>1 \mathrm{~g} / \mathrm{L}$, glucosa $<0,40 \mathrm{~g} / \mathrm{L}$, moderada pleocitosis mixta o de predominio linfocitario, que sólo ocasionalmente excede $\operatorname{los} 500 / \mathrm{mm}^{3}$, y adenosin deaminasa $(\mathrm{ADA})>7 \mathrm{U} / \mathrm{L})$.

Clasificación del déficit neurológico inicial. Se aplicó la escala descrita por Medical Research Council ${ }^{11,12}$ : Estadio 1: Consciente, sin signos neurológicos focales; Estadio 2: Confuso o con déficit neurológico focal; Estadio 3: Alguno de los siguientes hallazgos: sopor o coma, parálisis múltiples de nervios craneales, hemiplejía o paraplejía.

\section{Resultados}

Distribución por edad y sexo. El rango de edad fue de 21 a 79 años, con un promedio de 41 y una mediana de 39 años. Catorce (27\%) pacientes eran mujeres y 39 (74\%) hombres.

Co-morbilidades. En 9 (17\%) pacientes existía el antecedente remoto de TBC, 13 (25\%) presentaban coinfección con VIH, 11 (21\%) tenían etilismo crónico, 4 $(7,5 \%)$ diabetes mellitus y en 16 pacientes $(30 \%)$ no existían antecedentes mórbidos de significación.

Manifestaciones clínicas. El 74\% (39/53) de los pacientes tenía entre 7 y 21 días de evolución previa al ingreso y 13\% (7/53) presentaba más de 30 días de evolución. El rango osciló entre 1 y 120 días de evolución previa, con una mediana de 11 días.

La tríada sintomática de cefalea, fiebre y vómitos estaba presente en $30 \%(16 / 53)$ de los pacientes al ingreso, fiebre en $76 \%(40 / 53)$ y cefalea en $68 \%(36 / 53)$ (Tabla 1).

El 66\% (35/53) presentaba algún grado de compromiso de conciencia, estadio 1 en $22 \%$ (13/53), estadio 2 en $51 \%(28 / 53)$ y estadio 3 en $27 \%(12 / 53)$. Ochenta por ciento (42/53) presentaba signos meníngeos y $19 \%$ (10/53) tenía déficit neurológico focal (Tabla 1).

Hallazgos de laboratorio. Sesenta y ocho por ciento $(36 / 53)$ de los pacientes tenía en el LCR de ingreso proteinorraquia sobre $1,1 \mathrm{~g} / \mathrm{L}, 80 \% \quad(42 / 53)$ glucosa $<0,4 \mathrm{~g} / \mathrm{L}$, en $93 \%$ el recuento de leucocitos era $<500 / \mathrm{mm}^{3}$ (49/53), con predominio de mononucleares en $72 \%$ (38/ 53) de los casos (Tabla 2).

\begin{tabular}{|lcc|}
\hline \multicolumn{3}{|c|}{ Tabla 1. Frecuencia de síntomas y signos en } \\
meningitis TBC. (n = 53)
\end{tabular}

\begin{tabular}{|c|c|}
\hline Características de LCR & $\%$ \\
\hline \multicolumn{2}{|l|}{ Proteínas $(g / L)$} \\
\hline $0-0,3$ & 6 \\
\hline $0,31-1,0$ & 28 \\
\hline $1,1-3,0$ & 60 \\
\hline$>3,0$ & 6 \\
\hline \multicolumn{2}{|l|}{ Glucosa $\quad(g / d l)$} \\
\hline$<0,2$ & 43 \\
\hline $0,21-0,4$ & 34 \\
\hline$>0,41$ & 23 \\
\hline \multicolumn{2}{|l|}{ Células $/ \mathrm{mm}^{3}$} \\
\hline $5-50$ & 23 \\
\hline $51-200$ & 43 \\
\hline $201-500$ & 25 \\
\hline $501-1.000$ & 6 \\
\hline$>1.000$ & 3 \\
\hline \multicolumn{2}{|l|}{$\% \mathrm{MN}^{*}$} \\
\hline $0-25$ & 8 \\
\hline $26-50$ & 6 \\
\hline $51-75$ & 11 \\
\hline $76-100$ & 75 \\
\hline *Mononucleares & \\
\hline
\end{tabular}


El cultivo de Koch de LCR fue positivo en 45\% (24/ 53) de los pacientes y la baciloscopia en LCR fue negativa en $100 \%$. En la mayoría de los enfermos se dispuso de una sola muestra para realizar estos exámenes.

El valor de ADA en LCR inicial fue $>7 \mathrm{U} / \mathrm{L}$ en $80 \%$ (42/53) de los casos.

Características radiológicas. En 44\% (23/53) de los casos la radiografía de tórax de ingreso contribuyó al diagnóstico de TBC: en 23\% (12/53) era sugerente de TBC activa, en 19\% (10/53) tenía un patrón de TBC miliar y en un caso (2\%) había imágenes secuelares de TBC.

Tratamiento antituberculoso. Todos los pacientes que recibieron tratamiento anti-TBC se sujetaron a la normativa vigente del Ministerio de Salud (MINSAL) de Chile. Así, 49\% (26/53) de los pacientes recibió la asociación de isoniacida, rifampicina, pirazinamida y etambutol, administrados por vía oral, en una dosis diaria, durante 50 días, y luego isoniacida más rifampicina administrados dos veces por semana, hasta enterar 56 dosis. En 40\% (21/53) de los casos se sustituyó el etambutol por estreptomicina (normativa anterior).

No se inició tratamiento anti-TBC en 2 pacientes que fallecieron dentro de los 5 primeros días de hospitalización.

Recibieron corticosteroides sistémicos 87\% (46/53) de los pacientes. En los 4 casos sin corticoterapia, la letalidad fue más alta (11\% con corticoterapia versus $67 \%$ sin ella).

Reacción adversa atribuida a fármacos anti-TBC se presentó en 3 pacientes ( 2 hepatitis y 1 rash alérgico), que se mejoraron después de suspender la rifampicina.

Evolución clínica. El 63\% (33/53) de los pacientes presentó evolución favorable y en $23 \%$ (12/53) de los casos se observó la expresión de secuelas neurológicas (Tabla 3).

Complicaciones. En 28\% (15/53) de los pacientes se observó una complicación neurológica, en algunos más de una a la vez. Dentro de éstas destacaban por su frecuencia: hidrocefalia (diagnosticada por TAC de cerebro) en $23 \%$ (12/53), vasculitis (confirmada por

\begin{tabular}{|c|c|c|}
\hline Secuela & No pacientes & $\%$ \\
\hline Plejía de extremidades inferiores & 2 & 4 \\
\hline Paresia de extremidades & 7 & 13 \\
\hline Parálisis del VI par & 3 & 6 \\
\hline
\end{tabular}

TAC de cerebro en $60 \%$ de los pacientes) $13 \%$ (7/53), granuloma inflamatorio en $6 \%(3 / 53)$. El $87 \%$ de las complicaciones fue detectada entre los días 2 y 14 días de hospitalización.

Letalidad. La letalidad atribuible a MTBC fue de $17 \%$ (9 casos); el deceso se produjo en los 15 primeros días de hospitalización en 64\% (7/53) de los casos. En dos pacientes ocurrió entre los 16 y los 30 días, y un paciente falleció a los 106 días a consecuencia de una hidrocefalia.

Co-infección con VIH. La co-infección con VIH estaba presente en $30 \%(16 / 53)$ de los pacientes, en 13 de estos el diagnóstico de infección por VIH/SIDA precedió a la MTBC y en 3, el diagnóstico de ambas infecciones fue simultáneo. Dos casos estaban recibiendo terapia anti-retroviral. El 87\% (14/16) de los coinfectados era de sexo masculino. Sólo en 9 pacientes se conocía el recuento de linfocitos CD4, con un rango de 10 a 563 céls $/ \mathrm{mm}^{3}$ y la mediana de 94 céls $/ \mathrm{mm}^{3}$.

El cultivo de Koch en LCR fue positivo en $25 \%$ (4/16) de los pacientes y el valor de ADA en $\mathrm{LCR}>7 \mathrm{U} / \mathrm{L}$ se presentó en $87 \%$ (14/16) de los casos, con una mediana de $14 \mathrm{U} / \mathrm{L}$.

La letalidad en los pacientes co-infectados fue de $31 \%(5 / 16)$ y en el resto la evolución fue satisfactoria.

Todos los casos de reacción adversa atribuible al tratamiento anti-TBC se presentaron en pacientes coinfectados por VIH.

\section{Discusión}

Estudios recientes realizados en los países desarrollados han demostrado que la MTBC es un problema vigente. Sin embargo, la mayoría de estas series incluyen pacientes pediátricos y regímenes anti-tuberculosos heterogéneos ${ }^{13}$. Nuestra casuística incluye 53 pacientes adultos con MTBC, todos tratados en el Hospital de Enfermedades Infecciosas Dr. Lucio Córdova, centro de referencia nacional para esta patología. Destacamos que el tratamiento anti-TBC de este hospital se basa en las normas del Ministerio de Salud de Chile, que contempla un régimen de cuatro fármacos (isoniacida, rifampicina, pirazinamida y etambutol) más su aplicación observada y controlada.

En esta experiencia la mediana de edad fue de 39 años, hallazgo que se correlaciona con una máxima expresión clínica -descrita en la literatura médica- entre los 35 y 39 años $^{14-16}$.

En términos morfológicos, la MTBC es una meningoencefalitis que compromete principalmente las meninges basales, pero también el parénquima cerebral, las arterias y venas cerebrales. El cuadro clínico está condicionado por dichas alteraciones patológicas y, dependiendo de qué manifestaciones clínicas predomi- 
nen, se han descrito varias formas de presentación; la forma meníngea es la más frecuente y fácil de diagnosticar $^{17}$. El síndrome meníngeo puede estar ausente al ingreso en 10 a $30 \%$ de los casos, y así ocurrió en $20 \%$ de nuestros pacientes. La focalización neurológica (presente en 19\% al ingreso en nuestra serie) creó dificultad para el diagnóstico diferencial con abscesos cerebrales o accidentes vasculares, y la concomitancia de patologías, como alcoholismo y diabetes mellitus, pudo inclinar el diagnóstico hacia esas etiologías.

Una ayuda importante en la orientación diagnóstica cumple la radiografía de tórax ${ }^{18}$, que en nuestro estudio contribuyó al diagnóstico de infección tuberculosa en $44 \%$ de los casos.

Otro pilar fundamental en el diagnóstico es el análisis del LCR. La mayoría de los pacientes presenta alteraciones citoquímicas sugerente de meningitis linfocitaria, con proteína alta y glucosa baja. En esta serie clínica, 20\% de los casos no presentó estas características, situación que puede ocurrir en el estudio de la primera muestra de LCR; en estos casos se aconseja repetir la punción lumbar $2,4,15,17-19$.

El cultivo de Koch del LCR es el patrón de oro para el diagnóstico de $\mathrm{MTBC}^{4,8}$. Sin embargo, el bacilo de Koch es un microorganismo de lento crecimiento que puede tardar hasta 8 semanas para desarrollarse en un medio sólido ${ }^{20}$; así, aunque $45 \%$ de nuestros pacientes tuvieron cultivos positivos, ello no fue de utilidad para el diagnóstico precoz. El rendimiento de esta serie es similar al promedio nacional que no supera el $50 \%{ }^{15}$. Experiencias extranjeras han demostrado que la positividad del cultivo de Koch en el LCR sube con varias muestras sucesivas (generalmente 3 ) hasta $75 \mathrm{u}$ $80 \%$, aunque las últimas dos se obtengan después de iniciado el tratamiento empírico, ya que éste no debe postergarse ${ }^{19-21}$. En nuestro medio, generalmente se solicita cultivar sólo la primera muestra, comprometiendo probablemente la oportunidad de recuperar el Mycobacterium. La baciloscopia no fue útil para el diagnóstico, debido a su baja sensibilidad, situación que se describe en revisiones previas ${ }^{4,15,22}$.

La determinación de la actividad de ADA en LCR resultó ser una herramienta útil para el diagnóstico rápido de $\mathrm{MTBC}$, con una sensibilidad mayor que la baciloscopia ${ }^{21-23}$. En esta revisión el ADA fue $>7 \mathrm{U} / \mathrm{L}$ en $80 \%$ de los LCR de ingreso.

Tratando de optimizar el diagnóstico rápido de MTBC se han desarrollado algunos nuevos métodos, como son serología (ELISA) para detectar anticuerpos específicos y RPC. Esta segunda es una técnica compleja y difícil de estandarizar; existen dos métodos disponibles comercialmente: Gen-Probe y el test AMPLICOR M. tuberculosis ${ }^{7}$. En LCR eventualmente podría ser útil en caso de sospecha clínica de MTBC; sin embargo, la sensibilidad y especificidad en líquidos estériles no está bien determinada y tiene alto índice de falsos negativos. Debe tenerse en cuenta que este método es incapaz de distinguir entre microorganismos viables y no viables, por lo que no debe ser usado en la monitorización del tratamiento ${ }^{1,4,6,17,26}$.

El tratamiento anti-tuberculoso se inició en forma empírica, en la mayoría de los casos, antes de los cinco primeros días de hospitalización. El 87\% de los pacientes recibió además corticoterapia.

Destacamos que $78 \%$ de los pacientes presentaba compromiso de conciencia y/o signología neurológica focal al ingreso, hecho que se correlaciona con un peor pronóstico de la enfermedad. A pesar de esto la letalidad fue de $17 \%$, cifra inferior a la descrita con anterioridad en la literatura científica ${ }^{11,15,18}$. Podríamos atribuir esta baja letalidad al inicio precoz de la terapia específica y al uso de corticoterapia. Diversos estudios han puesto en evidencia que el tratamiento antituberculoso precoz y simultáneo con dexametasona mejora la sobreviva de pacientes con MTBC, sobre 14 años de edad, en forma independiente de la severidad de la enfermedad ${ }^{1,22-24}$.

Las complicaciones neurológicas ocurrieron en $28 \%$ de los pacientes, porcentaje menor a lo descrito en la literatura médica ${ }^{11,17,25}$, siendo la hidrocefalia la principal. Los procedimientos neuro-radiológicos son de gran utilidad en la detección precoz de estas complicaciones y en la evaluación de su tratamiento.

En nuestra serie es destacable que $30 \%$ de pacientes estaban co-infectados con VIH, en concordancia con el alto riesgo de desarrollo de $\mathrm{TBC}$ en este grupo de pacientes. Se ha descrito un aumento sobre $20 \%$ de las tasas de tuberculosis en co-infección con VIH, en regiones en que la prevalencia de la TBC es alta $6,18,26,27$. En sólo 9 de 16 pacientes se conocía el recuento de linfocitos CD4, destacando que su mediana fue menor a 100 células $/ \mathrm{mm}^{3}$, lo que se correlaciona con el mayor riesgo de desarrollar TBC extrapulmonar, especialmente MTBC, a medida que disminuye el recuento de CD4 en los pacientes con SIDA ${ }^{4,13,34}$. Las características clínicas y de laboratorio fueron en todo similar a los no infectados ${ }^{34,35}$. Cabe destacar que la letalidad fue mayor en este grupo de pacientes, 31 versus $17 \%$ del total de la muestra, lo que coincide con lo publicado ${ }^{6,12,34}$. Todas las reacciones adversas atribuibles al tratamiento anti-TBC se presentaron en este grupo de pacientes: 2 hepatitis y 1 rash alérgico, que se mejoraron después de suspender la rifampicina. Hoy día se considera que rifampicina es poco hepatotóxica, y raras veces produce hepatitis cuando no se está usando en forma concomitante isoniazida; esta última no es hepatotóxica per se, pero sí lo son algunos de sus derivados, especialmente la acetil hidrazina. La rifampicina es un pode- 
roso inductor del citocromo $\mathrm{P} 450$, y estimula el metabolismo de la isoniazida hacia sus derivados tóxicos para el hígado. De tal manera que el médico debe ser cauteloso al buscar la asociación de rifampicina con hepatotoxicidad en estos casos.

En conclusión, destacamos que la MTBC continúa estando presente y ha experimentado un incremento en su incidencia en estas últimas décadas a consecuencia de la pandemia del VIH. El diagnóstico de certeza precoz de esta patología no es posible con los métodos actuales, por lo que la sospecha clínica sigue siendo importante. Los estudios han demostrado que la precocidad en el inicio del tratamiento específico es determinante en la morbi-mortalidad de esta patología, por lo que es crucial tener elementos que orienten al diagnóstico precoz; son pilares fundamentales: las manifestaciones clínicas (antecedentes de enfermedad tuberculosa o clínica actual sugerente de tuberculosis), antecedente de contacto epidemiológico, la radiografía de tórax y el estudio de LCR.

\section{Resumen}

La meningitis tuberculosa (MTBC) es la forma más grave de tuberculosis extrapulmonar. El amplio espec- tro del cuadro clínico y su poca especificidad difícultan su diagnóstico precoz, relacionándose directamente con mayor letalidad. El objetivo de este estudio es analizar las características clínicas de los pacientes con diagnóstico de MTBC en el Hospital de Enfermedades Infecciosas Dr. Lucio Córdova, de Santiago, Chile, entre 1995 y 2002. Se estudiaron 53 casos de MTBC en adultos, con una edad mediana de 39 años. Al ingreso $66 \%$ de los pacientes presentaba algún grado de compromiso de conciencia, pero sólo $30 \%$ la tríada sintomática clásica. El LCR mostró aumento de proteínas, glucosa baja y linfocitosis, en la mayoría de los casos. Treinta por ciento de los pacientes presentó co-infección con VIH; la letalidad en este grupo de pacientes fue mayor, 31 vs $17 \%$ del total de la muestra. La MTBC continúa siendo una enfermedad vigente. El diagnóstico de certeza precoz de esta patología no es posible con los métodos actuales, por lo que la sospecha clínica es importante.

\section{Agradecimientos}

Agradecemos la cooperación de Aurora Garín D., Enna Zunino M. y Laura Bahamondes M., por el tiempo destinado a la revisión y discusión del estudio.

\section{Referencias}

1.- Twaites G, Duc Bang N, Huy Dung N, Thi Quy H, Thi Tuong D, Thi Cam N, et al. Dexamethasone for the treatment of tuberculous meningitis in adolescents and adults. N Engl Med 2004; 351: 1741-51.

2.- Kent S, Crowe S, Yung A, Lucas C, Mijch A. Tuberculous meningitis: A 30-year review. Clin Infect Dis 1993; 17: 987-94.

3.- Donald P, Schoeman J. Tuberculous meningitis. N Eng J Med 2004; 351: 171920 .

4.- Thwaites G, Chau T T H, Mai N T H, Drobniewski F, McAdam K, Farrar J. Tuberculous meningitis. J Neurol Neurosurg Psychiatry 2000; 68: 289-99.

5.- Girardi E, Raviglione M C, Antonucci G, Godfrey-Faussett P, Ippolito G. Impact of the HIV epidemic on the spread of other diseases: the case of tuberculosis. AIDS 2000; 14 (Suppl 3): 47-56.

6.- Zumla A, Malon P, Henderson J, Grange J. Impact of HIV infection on tuberculosis. Postgrad Med 2000; 76: 259-68.

7.- Havlir D, Barnes P. Tuberculosis in patients with human immunodeficiency virus infection. N Engl J Med 1999; 340: 367-73.

8.- Twaites G E, Chau T, Phu N, Choung L, White N J, Parry C et al. D. Diagnosis of adult tuberculous meningitis by use of clinical laboratory features. Lancet 2002; 360: 1287-92.

9.- Streptomycin in Tuberculous Trials Committee, Medical Research Council. Streptomycin treatment of tuberculous meningitis. Lancet 1948; 1: 582-96.

10.- Fitzgerald D, Haas D. Mycobacterium tuberculosis. Mandell, Douglas and Bennett's Principles and Practice of Infectious Diseases, Mandell GL, Bennett JE, Dolin R, editors. Sixth ed. Philadelphia: Churchill Livingstone; 2005, p. 2852-86.

11.- Verdon R, Chevret S, Laissy J P, Wolff M. Tuberculous meningitis in adults: Review of 48 cases. Clin Infect Dis 1996; 22: 982-8.

12.- Braun M, Coté T, Radkin Ch. Trends in death with tuberculosis during the AIDS era. JAMA 1993; 269: 2865-8.

13.- García-Martos P, Castaño M A, Canueto J, Hohr L, González-Borrachero M L. Tuberculosis en 34 pacientes con infección por VIH. Enferm Infec Microbiol Clin 1991: 64-6.

14.- Yang Z, Kong Y, Wilson F, Foxman B, Fowler A, Marrs C, et al. Identification of risk factors for extrapulmonary tuberculosis. Clin Infect Dis 2004; 38: 199-205.

15.- Garin A, Bahamondes L, Hojas R. Meningitis tuberculosa. Análisis clínico de 42 enfermos. Rev Chil Infect 1989; 6: 205-11.

16.- Abdel-Dayem H, Naddaf S, Asís M, Mina B, Turoglu T, Akisik M et al. Sites of tuberculous involvement in patients with AIDS. Autopsy findings and evaluation of gallium imaging. Clin Nuclear Med 1997; 22: 310-4.

17.- Girgis N, Sultan Y, Farid Z, Manssur M, Erian M, Hanna L et al. Tuberculous meningitis; Abbassia Fever Hospital-Naval Medical Research Unit NO.3- Cairo, Egypt, from 1976-1996. Am Trop Med Hyg 1998; 58: $28-34$.

18.- Arzuaga J, de la Fuente J, Pérez R, Masa C, Martinez J, Letona M. Meningitis tuberculosa en pacientes no infectados por VIH. Presentación de 21 casos. Enferm Infec Microbiol Clin 1992; 10: 576-80. 
19.- Karen L R, Kenneth L T. Meningitis, encephalitis, brain abscess and empyema. En Harrison's Principles of Internal Medicine, Kasper et al. 16th Edition 2005; pp: 2471-82.

20.- Baker C, Catwright P, Williams S, Nelson S, Peterson P. Early detection of central nervous system tuberculosis with the GenProbe Nucleic Acid Amplication assay: Utility in an inner City Hospital. Clin Infect Dis 2002; 35: 339-42.

21.- López-Cortés L F, Cruz-Ruiz M, Gómez-
Mateos J, Jiménez-Hernández D, JiménezMejias E, et al. Adenosine deaminase activity in the CSF of patients with aseptic meningitis: Utility in the diagnosis of tuberculous meningitis or neurobrucellosis. Clin Infect Dis 1995; 20: 525-30.

22.- Dooley D, Carpenter J, Rademager S. Adjuntive corticosteroid therapy for tuberculosis: A critical reappraisal of the literature. Clin Infect Dis 1997; 25: 872-87.

23.- Ramos JM, Esteban J, Fernández-Guerrero M L, Soriano F. Meningitis tuberculosa: aspectos pronósticos de 22 casos con confirmación microbiológica. Enferm Infec Microbiol Clín 1995; 13: 12-6.

24.- Berenguer J, Moreno S, Laguna F, Vicente T, Adrados M, Ortega A, et al. Tuberculous meningitis in patients infected with human immunodeficiency virus. N Engl J Med 1992; 326: 668-72.

25.- Dube M, Holtom P, Larsen R. Tuberculous meningitis in patients with and without human immunodeficiency virus infection. Amer J Med 1992; 93: 520-4. 